\title{
An Improved Fuzzy Controlled Asynchronous Transfer Mode (ATM) Network
}

\author{
C. IHEKWEABA and G.N. ONOH
}

\begin{abstract}
This paper presents basic features of the Asynchronous Transfer Mode (ATM). It further showcases Fuzzy Logic as an effective system control mechanism. MATLAB; the simulation kit used in the development of the system is described. The models that yielded high performance values are shown. Finally, the test results and their implications are ellucidated.
\end{abstract}

Index Terms-Asynchronous Transfer Mode, Constant Bit Rate, Variable Bit Rate, Matlab, Fuzzy logic.

\section{INTRODUCTION}

Asynchronous Transfer Mode (ATM)[1,3,7] also referred to as cell switching utilizes the concept of Virtual Circuit Switching [2,18].

It consists of a Fifty Three (53) bytes fixed packet, which is used to transfer information simultaneously from either voice, data or video sources [19].

ATM has the ability to provide seamless networking $[3,17]$ as well as a universal networking platform.

Various Quality of service (QOS) parameters can be negotiated on an ATM network. They include call Delay variables; Maximum Cell Transfer Delay (max CTD), Peak to Peak cell Delay Variation (P2P-CDV), Cell Error Ratio (CER), Cell Misinsertion Ratio (CMR) and Severely Errored Cell Block ratio (SECB).

Various classes of ATM services guarantee different Quality of service and traffic parameters, which include; Constant Bit Rate (CBR), Real time Variable Bit rate (rtVBR), Non- real time Variable Bit rate (nrt-VBR), Unspecified Bit rate (UBR), Available Bit rate (ABR) and Guaranteed Frame rate (GFR)[5]. ATM simultaneously attempts to support voice, data and video applications, each one having different performance requirements: It thus becomes imperative that for optimal utilization of the network, the system architecture requires complex, non linear distributed control structures. In order to achieve it's potential, the ATM network will need to accommodate several interacting control mechanisms such as Call Admission Control,[4] Flow and Congestion Control[6,8], Input rate regulation, Routing, Bandwidth allocation, Queue Scheduling and Buffer management. It thus becomes necessary that a strategic system control architecture be employed in ATM Control. A Fuzzy logic control system was adopted in this work because of its robustness in the control of typical systems.

\section{FUZZY LOGIC CONTROL}

Fuzzy logic is a superset of conventional (Boolean) logic that has been extended to handle the concept of partial truth- truth values between "completely true" and completely false"[12]. As it's name suggests, it is the logic underlying modes of reasoning which are approximate rather than exact. The importance of Fuzzy logic derives from the fact that most modes of human reasoning and especially common sense reasoning are approximate in nature. Fuzzy logic is a problem-solving control system methodology, capable of generating conclusions based upon vague, ambiguous, imprecise, noisy or missing input information. This approach follows naturally how a professional is able to solve a problem.

Fuzzy logic incorporates a simple rule based IF, THEN statements rather than attempting to model the system.

Fuzzy logic is empirically based; it relies on the operators' experience rather than the technical details of the system being controlled. Expressions such as voltage is low, are common instead of voltage is $2 \mathrm{~V}$.

Fuzzy logic is currently preferred in control systems because it is robust and does not insist on noise- free inputs and can implement non linear systems without any known mathematical models. The output control is usually a smooth control function even when a wide range of input variations exist [10].

It is easier to modify the system for the purpose of either altering or improving it's performance, by changing the rule structure, rule base, membership function defuzzyfication process.

The cost of fuzzy system implementation is low. Since the system can easily be simulated before implementation.

Multiple inputs and outputs can be achieved with Fuzzy logic controlled systems[13]. The number of the signals being a major determinant of the complexity of the rule base.

Due to its capacity to capture human expertise and to formalize approximate reasoning processes, it is a veritable tool for handling the challenges of congestion control in ATM networks. The basic steps employed in Fuzzy logic implementation, involves identifying and defining the control objective, determining the input and output relationships, developing the rule base using simple IF, THEN, AND, OR operations, and determining the Fuzzy logic membership functions[14]. Subsequently, the necessary routines are created if the system is intended to be implemented in software. Otherwise, the rules are coded directly into the system for hardware based implementation.

Finally, the system is tested and in some cases tuned for optimum operation.

\section{MATLAB ${ }^{\circledR}$}

The simulation kit used in this work is MATLAB. The name stands for Matrix Laboratory. It is a high performance 
tool that enhances technical computing as it integrates computation, visualization and programming in a user friendly computer system environment[11].

MATLAB is a collection of increasingly Add-on Application specific solutions referred to as tool Boxes, which are a comprehensive collection of MATLAB functions referred to as $\mathrm{M}$-files, for signal processing, control systems, Fuzzy Logic, Genetic Algorithm, Neural Networks, Wavelets, Filter design simulations [15] etc.

\section{Assumptions}

The following assumptions were used in this work,

1. The Quality of service (QoS) and Traffic parameters are dynamic and therefore assume values that can correctly be modeled as random.

2. The values of the Linguistic input variables are normalized and have magnitudes within the range of 0 and 1

3. The threshold value as specified in the final output, determine whether a cell can be accepted or rejected. Therefore, an observation of the output of the controller, and reference to the value of the specified threshold, is a good indication of acceptance or rejection

4. The ratio of the number of cells accepted to those rejected, is an accurate measure of system efficiency.

\section{RESEARCH METHODOLOGY}

Different models of fuzzy controlled ATM systems were formulated. These models were simulated using the Fuzzy logic tool box on MATLAB release 7.0. As a model is proposed, various linguistic input variables and membership functions are developed. The attendant rule bases in accordance with the Mamdani rule structure are established based on experience and tendency of current results obtained. The entire systems were rigorously tuned, generating well over one hundred experiments. Also, a MATLAB program was developed and used to randomly generate one hundred (100) different input values for the linguistic variables prescribed. The program also counts and displays the number of cells accepted and also those rejected, which now served as a basis for efficiency as stipulated by the work.

\section{TESTS AND RESULTS}

Several models were developed with their attendant rule bases. The following, fig1, fig5, fig 10, fig14 are the models that yielded high results, their typical surface plots are shown in figs; 2,3,4,6,7,8,10,11,12,14,15 and 16. Plots describing the Acceptance/rejection pattern for each model are shown in figs. 5,9,13 and 17 respectively. The model III in fig3, with it's attendant rule base(Tables i, ii and iii) yielded the highest result. Table II is a summary of the results for all the models showcased.
Model 1

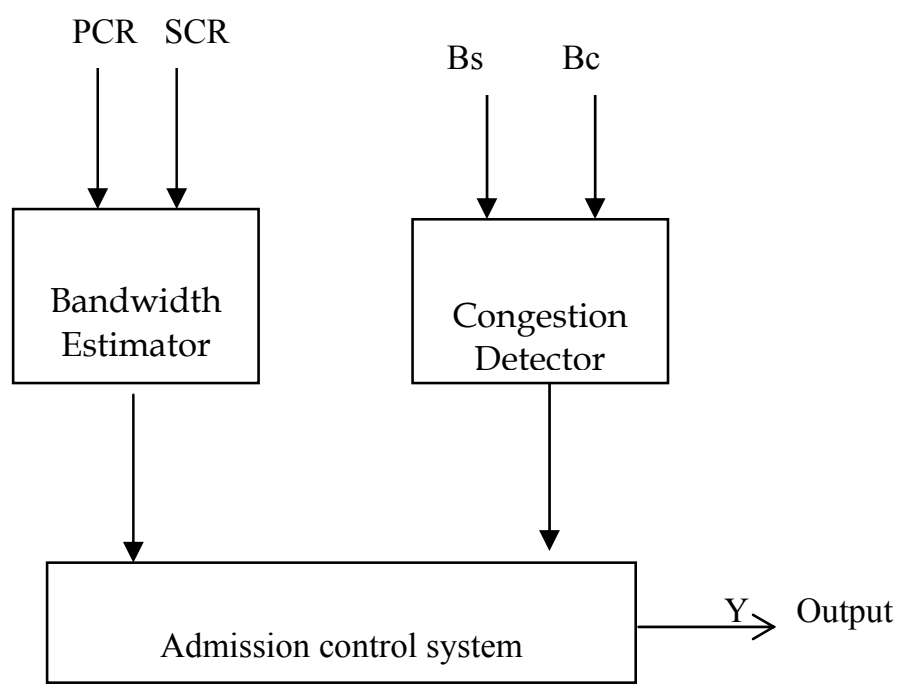

Fig 1 Model 1 of the fuzzy controlled ATM Network

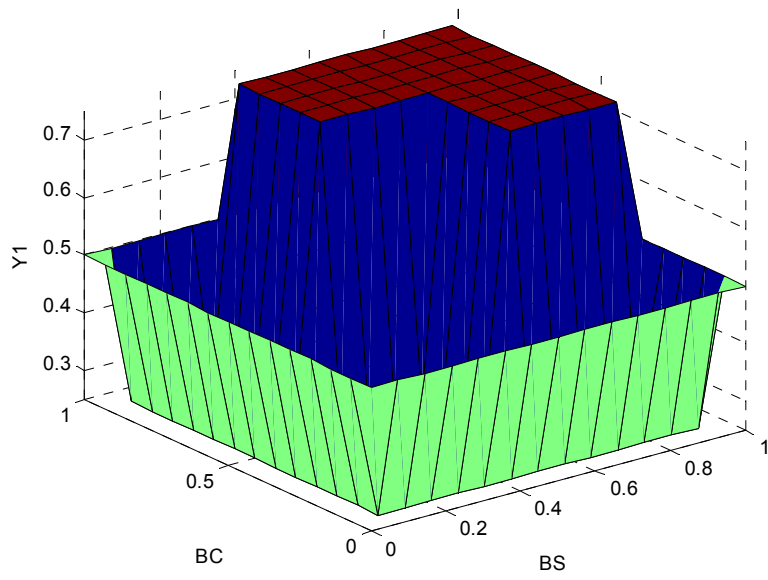

Fig 2. A surface plot for Model I of the Fuzzy Controlled ATM network

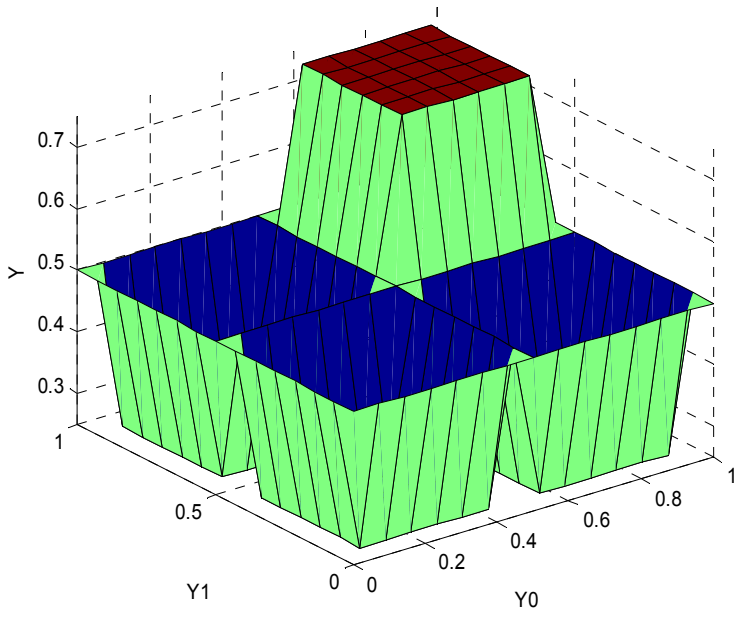

Fig 3. A surface plot for Model I of the Fuzzy Controlled ATM network 


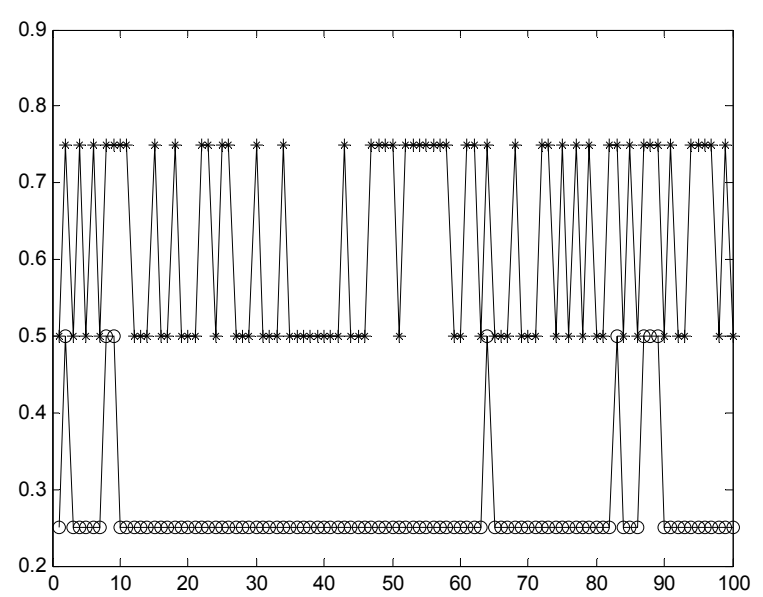

Fig 4 A Graph showing the Acceptance /Rejection ratio for Model I of the Fuzzy Controlled ATM network

\section{Model II}

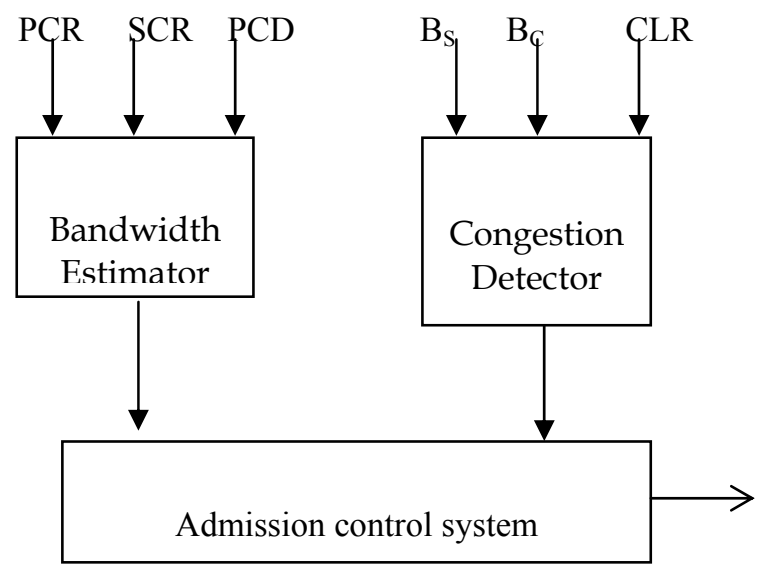

Fig 5 Model II of the fuzzy controlled ATM network

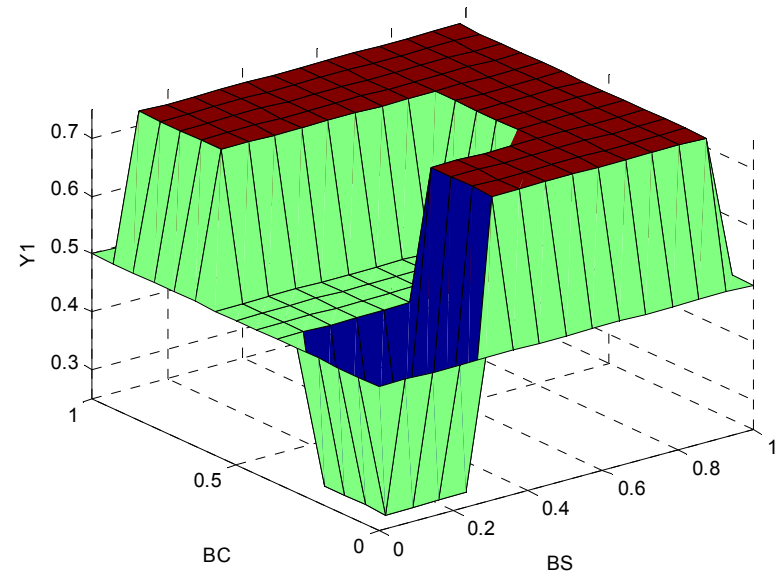

Fig 6 A surface plot for Model II of the Fuzzy Controlled ATM network

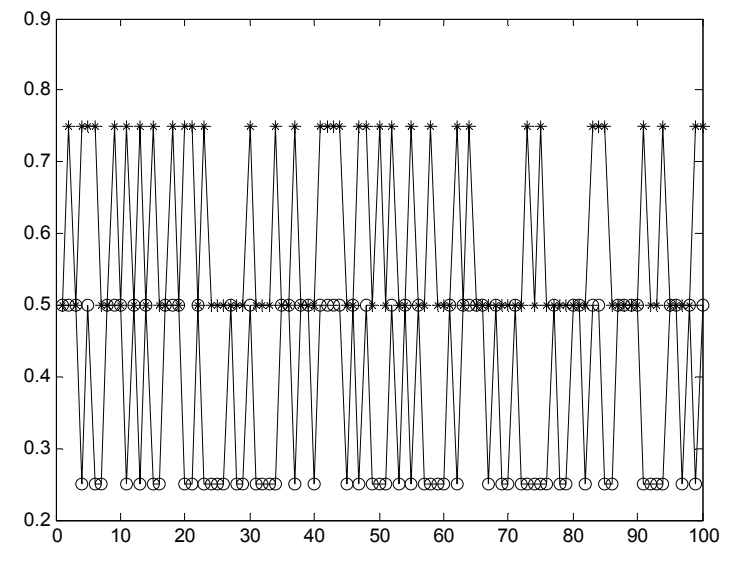

Fig 7 A Graph showing the Acceptance /Rejection Ratio for Model II of the Fuzzy Controlled ATM network

Model III

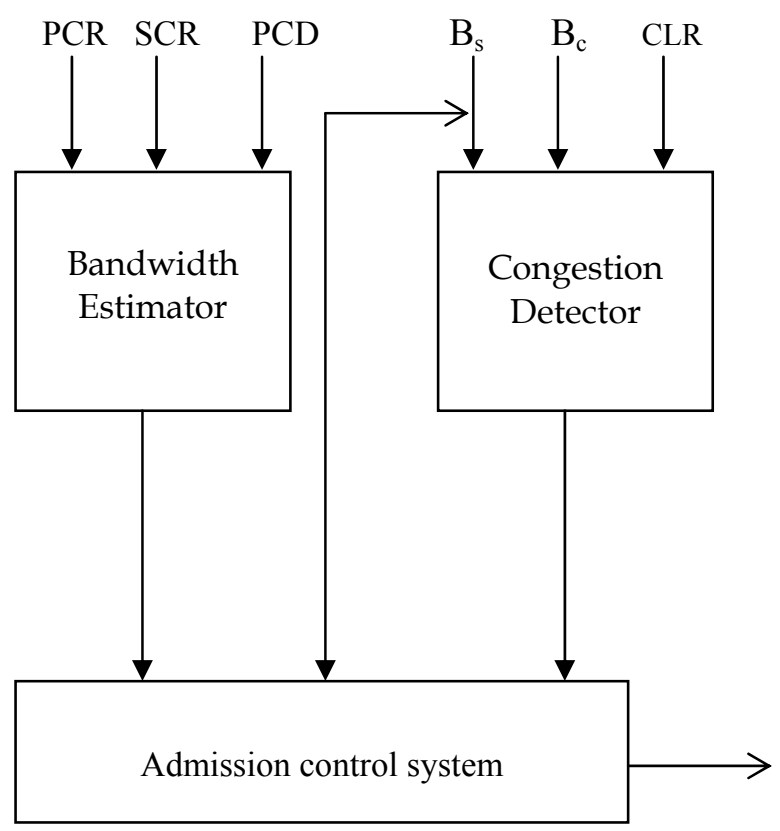

Fig 8 Model III of the fuzzy controlled ATM network

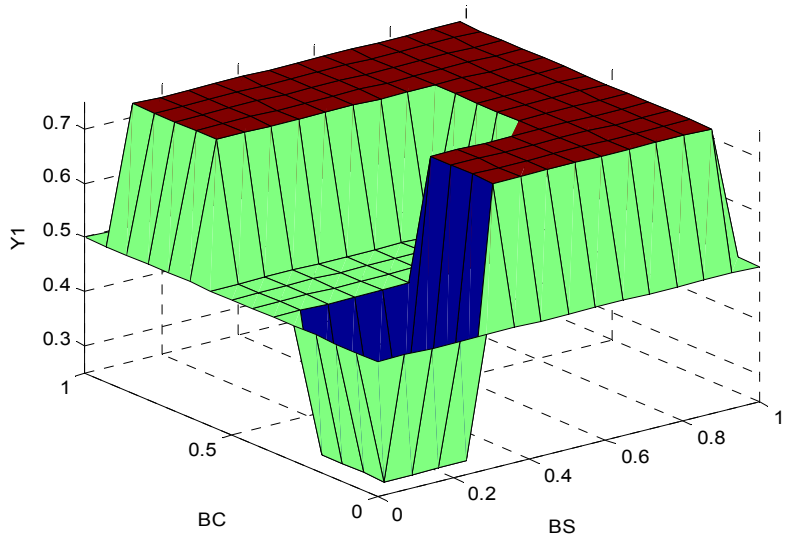

Fig 9 A surface plot for Model III of the Fuzzy Controlled ATM network 


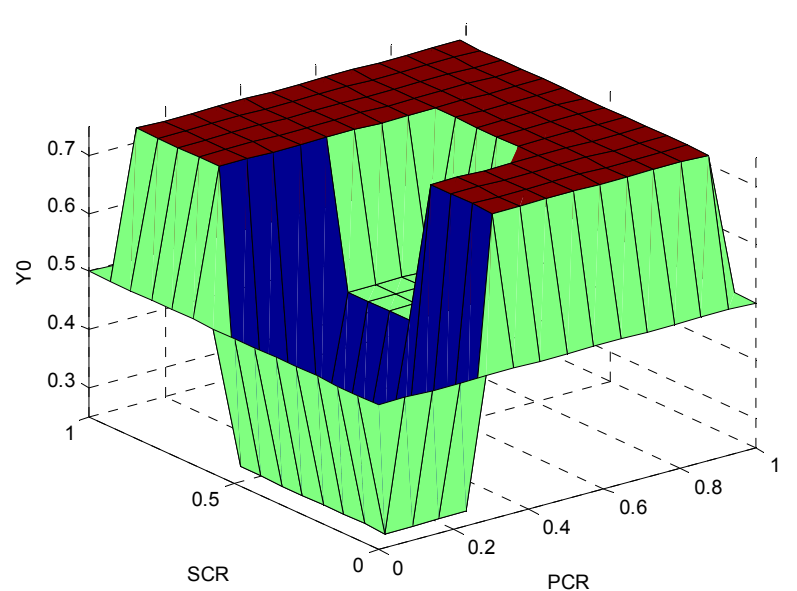

Fig 10 A surface plot for Model III of the Fuzzy Controlled ATM network

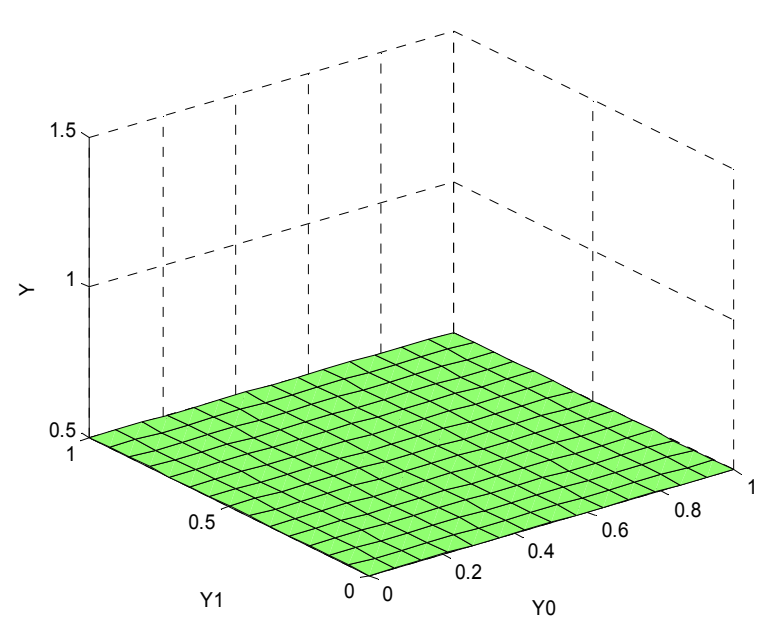

Fig 11 A surface plot for Model III of the Fuzzy Controlled ATM network

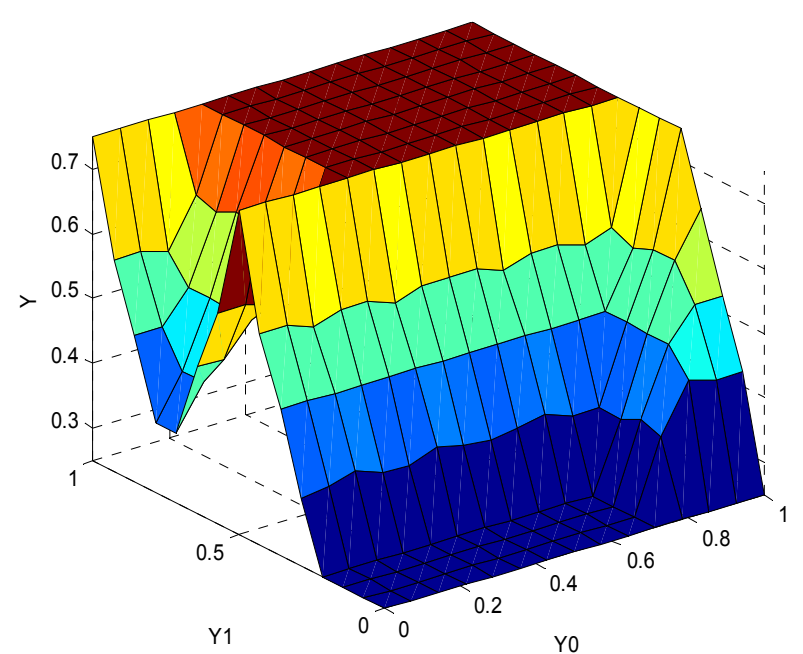

Fig 12 A surface plot for Model III of the Fuzzy Controlled ATM network

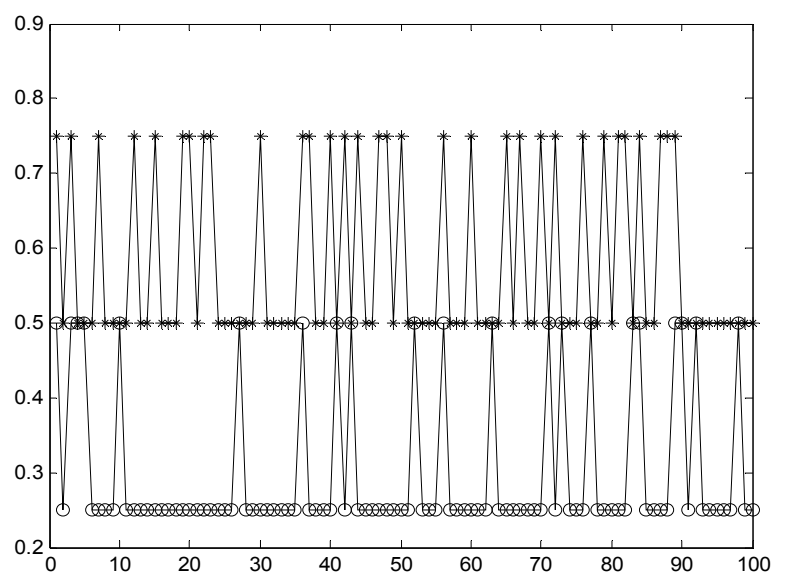

Fig 13 A Graph showing the Acceptance /Rejection Ratio for Model III of the Fuzzy Controlled ATM network

\section{Model IV}

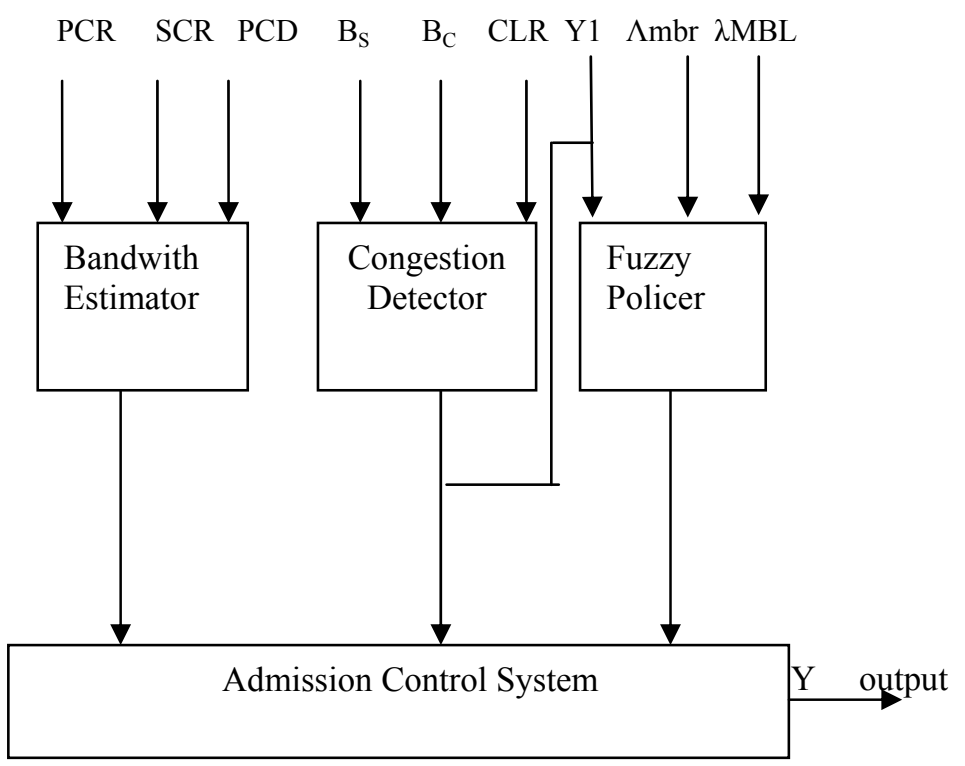

Fig 14 model IV of the Fuzzy Controlled ATM network

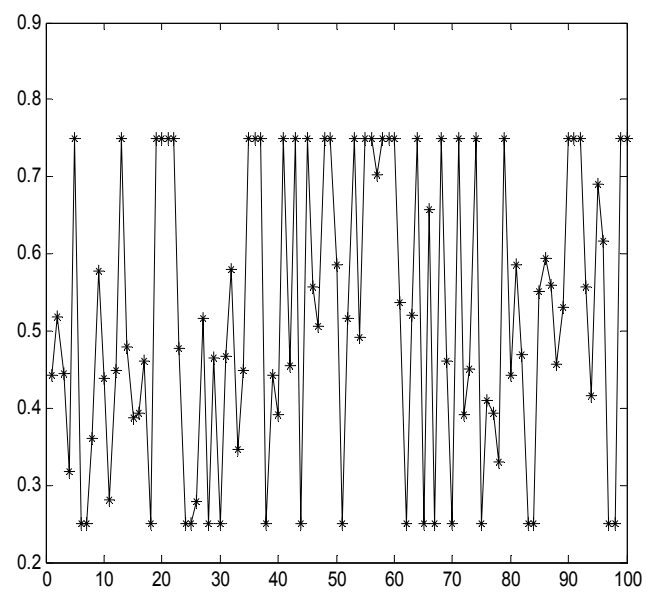

Fig 15 A Graph showing the Acceptance/Rejection Ratio for Model IV 
VI. THE RULE BASE FOR THE BANDWIDTH EsTIMATOR IS AS FOLLOWS

1. If PCR is small and SCR is small and PCD is small then $\mathrm{Y}_{0}$ is low

2. If PCR is small and SCR is small and PCD is medium then $\mathrm{Y}_{0}$ is low

3. If PCR is small and SCR is small and PCD is High then $\mathrm{Y}_{0}$ is low

4. If PCR is small and SCR is medium and PCD is small then $\mathrm{Y}_{0}$ is low

6. If PCR is small and SCR is medium and PCD is High then $\mathrm{Y}_{0}$ is low

7. If PCR is small and SCR is medium and PCD is small then $\mathrm{Y}_{0}$ is low

8. If PCR is small and SCR is High and PCD is medium then $\mathrm{Y}_{0}$ is high

9. If PCR is small and SCR is High and PCD is high then $\mathrm{Y}_{0}$ is high

10. If PCR is medium and SCR is High and PCD is small then $\mathrm{Y}_{0}$ is high

11. If PCR is medium and SCR is small and PCD is medium then $\mathrm{Y}_{0}$ is high

12. If PCR is medium and SCR is small and PCD is high then $\mathrm{Y}_{0}$ is low

13. If PCR is medium and SCR is small and PCD is small then $\mathrm{Y}_{0}$ is low

14. If PCR is medium and SCR is small and PCD is medium then $\mathrm{Y}_{0}$ is High

15. If PCR is medium and SCR is small and PCD is high then $\mathrm{Y}_{0}$ is low

16. If PCR is medium and SCR is high and PCD is small then $\mathrm{Y}_{0}$ is high

17. If PCR is medium and SCR is high and PCD is medium then $\mathrm{Y}_{0}$ is High

18. If PCR is medium and SCR is High and PCD is high then $\mathrm{Y}_{0}$ is High

19. If PCR is medium and SCR is Low and PCD is low then $\mathrm{Y}_{0}$ is High

20. If PCR is High and SCR is Low and PCD is medium then $\mathrm{Y}_{0}$ is High

21. If PCR is High and SCR is Low and PCD is high then $\mathrm{Y}_{0}$ is High
22. If PCR is High and SCR is medium and PCD is low then $\mathrm{Y}_{0}$ is High

23. If PCR is High and SCR is medium and PCD is medium then $\mathrm{Y}_{0}$ is High

24. If PCR is High and SCR is medium and PCD is high then $\mathrm{Y}_{0}$ is High

25. If PCR is High and SCR is High and PCD is low then $\mathrm{Y}_{0}$ is High

26. If PCR is High and SCR is High and PCD is medium then $\mathrm{Y}_{0}$ is High

27. If PCR High and SCR is High and PCD is High then $\mathrm{Y}_{0}$ is High

TABLE VII THE RULE BASE FOR THE BANDWIDTH ESTIMATOR FOR MODEL III

TABLE VII.

1. If BS is small and BC is small and CLR is small then $\mathrm{Y} 1$ is low

2. If BS is small and BC is small and CLR is medium then $\mathrm{Y} 1$ is low

3. If BS is small and BC is small and CLR is High then $\mathrm{Y} 1$ is low

4. If BS is small and BC is medium and CLR is small then $\mathrm{Y} 1$ is low

6. If BS is small and BC is medium and CLR is High then $\mathrm{Y} 1$ is low

7. If BS is small and BC is medium and CLR is small then $\mathrm{Y} 1$ is low

8. If BS is small and BC is High and CLR is medium then $\mathrm{Y} 1$ is high

9. If BS is small and BC is High and CLR is high then $\mathrm{Y} 1$ is high

10. If BS is medium and BC is High and CLR is small then $\mathrm{Y} 1$ is high

11. If BSis medium and BC is small and CLR is medium then $\mathrm{Y} 1$ is high

12. If BS is medium and BC is small and CLR is high then $\mathrm{Y} 1$ is low

13. If BS is medium and BC is small and CLR is small then $\mathrm{Y} 1$ is low

14. If BS is medium and BC is small and CLR is medium then Y1 is High 
15. If BS is medium and BC is small and CLR is high then $\mathrm{Y} 1$ is low

16. If BS is medium and BC is High and CLR is small then $\mathrm{Y} 1$ is High

17. If BS is medium and BC is high and CLR is medium then Y1 is High

18. If BS is medium and BC is High and CLR is high then $\mathrm{Y} 1$ is High

19. If BS is medium and BC is Low and CLR is low then Y1 is High

20. If BS is High and BC is Low and CLR is medium then $\mathrm{Y} 1$ is High

21. If BS is High and BC is Low and CLR is high then Y1 is High

22. If BS is High and BC is medium and CLR is low then Y1 is High

23. If $\mathrm{BS}$ is High and $\mathrm{BC}$ is medium and CLR is medium then $\mathrm{Y} 1$ is High

24. If BS is High and BC is medium and CLR is high then Y1 is High

25. If BS is High and BC is High and CLR is low then Y1 is High

26. If BS is High and BC is High and CLR is medium then $\mathrm{Y} 1$ is High

27. If $\mathrm{BS}$ High and $\mathrm{BC}$ is High and CLR is High then $\mathrm{Y} 1$ is High

\section{TABLE VIII THE RULE BASE FOR THE CONGESTION DETECTOR FOR MODEL III}

VIII The Rule Base for the Admission Control Syste

1.If $\mathrm{y}_{0}$ is low and $\mathrm{y}_{1}$ is Low and $\mathrm{y} 2$ is low

Then $\mathrm{y}$ is reject

2. If $\mathrm{y}_{0}$ is low and $\mathrm{y}_{1 \text { is Low }}$ and $\mathrm{y} 2$ is High Then $\mathrm{y}$ is reject

3.If $\mathrm{y}_{0}$ is low and $\mathrm{y}_{1}$ is High and $\mathrm{y} 2$ is low Then $\mathrm{y}$ is reject

4.If $\mathrm{y}_{0}$ is low and $\mathrm{y}_{1}$ is High and $\mathrm{y} 2$ is High Then $\mathrm{y}$ is Admit

5.If $\mathrm{y}_{0}$ is High and $\mathrm{y}_{1}$ is Low and $\mathrm{y} 2$ is low Then $\mathrm{y}$ is reject

6.If $\mathrm{y}_{0}$ is High and $\mathrm{y}_{1 \text { is Low }}$ and $\mathrm{y} 2$ is High Then y Admit
7.If $\mathrm{y}_{0}$ is High and $\mathrm{y}_{1}$ is High and $\mathrm{y} 2$ is low Then $\mathrm{y}$ is Admit

8.If $\mathrm{y}_{0}$ is High and $\mathrm{y}_{1}$ is High and $\mathrm{y} 2$ is High Then $\mathrm{y}$ is Admit

TABLE IX RULE BASE FOR THE ADMISSION CONTROL SYSTEM FOR MODEL III

\section{Membership Function}

Triangular

From 0 to 0.3 Small

From 0.4 to 0.6 Medium

From 0.6 to 1.0 High

VII. RESULTS

\begin{tabular}{|l|c|c|c|}
\hline & $\begin{array}{c}\text { Total No Of } \\
\text { Calls }\end{array}$ & No Accepted & No Rejected \\
\hline Model I & 100 & 55 & 45 \\
\hline Model II & 100 & 81 & 19 \\
\hline Model III & 100 & 87 & 13 \\
\hline Model IV & 100 & 62 & 38 \\
\hline
\end{tabular}

\section{CONCLUSION}

An effective controller for the ATM network is derived from a fuzzy controlled system that can effectively monitor the quality of service and traffic parameters to initially determine the bandwidth requirements of the cells, evaluate the current state of the network, use these status to determine the acceptability of a cell seeking permission for transmission. The system derived has an efficiency of $87 \%$.

\section{REFERENCES}

[1] William S, 2007, "Data and Communication", Prentice Hall of India pp298-323

[2] Sumit Kasera, 2006 "ATM Networks, Concepts and Protocols" Tata Magraw Hill India pp27

[3] Call Admission Control

[4] Sumit Kasera, 2006 "ATM Networks, Concepts and Protocols" Tata Magraw Hill India pp141

[5] William S, 2007 "Data and Computer Communications" PrenticeHall of India. pp 383-388

[6] Denis R, John C, 2004, “ Electronic Communication”, Pearson Education India pp 415.

[7] Beaucham K. G, 1990, “computer Communications”, Chapman and Hall London pp 115

[8] Forouzan B.A, 2003 "Data Communications and Networking" Tata McGraw-Hill India, pp447 - 449

[9] Mathworks Inc, 1999, "Fuzzy logic Toolbox users Guide", pp1 - 63.

[10] Hahn B.D and Valentine D.T, 2007, "Essential MATLAB for Engineers and Scientists", Elsevier Ltd, pp1 - 103.

[11] Matworks Inc, 2004, "Genetic Algorithm and Direct Search Toolbox for use with MATLAB pp $1-120$.

[12] http://www.lpa.co.uk/fln.htm?gclid=CL60pZzG7J8CFdx05Qod8Rydg

[13] http://en.wikipedia.org/wiki/Fuzzy_logic

[14] http://www.seattlerobotics.org/Encoder/mar98/fuz/fl_part1.html 


\section{BIOGRAPHY}

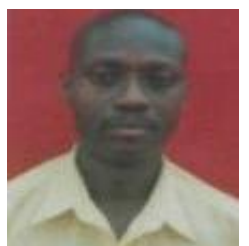

Ihekweaba Chukwugoziem holds a Bachelors degree in Electrical/Electronics Engineering Anambra State University of Technology, Enugu, Nigeria in 1987. A Masters degree in Computer Science and Engineering from the same University in 1990. A Doctorate degree in Communication \& Engineering, Enugu State University of Science and Technology Enugu, Nigeria. The Author's major field of study is Application of Computational Intelligence in Data Communication Networks.

He has been involved in several projects in Data Communications and has taught Computer Science and Engineering since 1987. He has presented papers in several conferences, published articles in several journals and three books in wide use in several higher institutions.

Engr. Ihekweaba is a member of Nigeria Society of Engineering (NSE), Council for regulation of Engineering in Nigeria (COREN), Nigeria Computer Society (NCS) and Computer Professionals of Nigeria (CPN).

He is currently the Acting Head, Department of Computer Engineering, Michael Okpara University of Agriculture, Umudike. 